\title{
Caisse de secours des médecins suisses
}

\author{
Rapport annuel 2001
}

En 2001, la Caisse de secours des médecins suisses a accordé son soutien à 30 médecins tombés malgré eux dans le besoin, ainsi qu'aux veuves, descendants et proches de consœurs et de confrères décédés.

Au cours de l'année dernière, deux nouveaux cas sont venus s'ajouter à la liste des bénéficiaires, pour lesquels l'aide de la Caisse se limitera probablement à l'année passée sous revue. En revanche, la Caisse a cessé d'apporter son soutien dans trois autres cas en raison de l'amélioration de leur situation. Aucun décès n'est heureusement à déplorer en 2001 parmi les bénéficiaires. Au 31 décembre 2001, leur nombre s'élevait encore à 26 personnes, soit une de moins que l'année précédente et la somme globale des contributions avait diminué, passant de Fr. 306 000.- en 2000 à Fr. 296 600.- en 2001.

Dans le respect strict des buts de la fondation, nous avons veillé comme toujours à la répartition la plus efficace possible des fonds à disposition.

Il est vrai que dans bien des situations, nous souhaiterions pouvoir fournir une aide plus substantielle, mais la limite supérieure des montants octroyés a dû être fixée impérativement, les moyens de la Caisse de secours étant par la force des choses limités.

Après la baisse de l'année précédente, le produit de la récolte de fonds 2001 a connu fort heureusement une augmentation. De plus, grâce à la politique de placement judicieuse de notre administrateur, qui a su réaliser des gains en capital au bon moment, la fortune de la Fondation s'est notablement accrue, et cela en dépit d'une situation boursière très difficile. Cependant, vu la persistance de l'instabilité économique, une partie importante du bénéfice a été dévolue à des provisions pour se prémunir contre la volatilité des titres.

Comme cité précédemment, les dons ont notablement augmenté en 2001, sans couvrir toutefois les dépenses courantes pour nos bénéficiaires. De Fr. 124425.30 en 2000, ils ont passé à Fr. 178119.85 pour 2001. Il ne reste plus qu'à espérer que cette tendance à la hausse s'affermira.

Les contributions modestes forment l'essentiel du capital de notre fondation. Par souci d'économie, nous renonçons toutefois à remercier personnellement les donateurs. Qu'ils veuillent trouver ici l'expression de notre vive gratitude.

Nos recettes pour l'année 2001, qui s'élèvent à Fr. 645 786.97, proviennent pour 30\% des dons et des honoraires pour attestations établies par les médecins et pour $70 \%$ du rendement et du bénéfice en capital, cela - disons-le encore une fois - grâce à une politique de placement à la fois judicieuse et prudente.

Durant l'exercice écoulé, environ $45 \%$ des fonds récoltés provenaient de dons individuels de Fr. 500.- et plus, ainsi que des versements de la FMH, de sociétés cantonales de médecine, de groupements médicaux et de commissions de déontologie. Nous avons également reçu deux legs particuliers, l'un de Fr. 3000.- et l'autre de Fr. 5000.--

Au 31 décembre 2001, la fortune de la fondation s'élevait à Fr. 2615142.89 . M. F. Hufschmid et le Dr R. Jucker, réviseurs, ont examiné les comptes annuels de la Caisse de secours et constaté que la comptabilité était tenue dans les règles. Pour plus de détails, voir les comptes et le bilan 2001 ci-devant.

Nous saisissons l'occasion pour exprimer une fois de plus notre vive reconnaissance aux autres membres du conseil, les Drs Ludwig Heuss et Urs Leibundgut ainsi qu'à MM. J. Kobler et W. Mahrer de la Banque La Roche \& Cie pour la tenue de la comptabilité et la gestion de fortune. Une fois de plus, il est réjouissant de souligner l'active collaboration de toutes les personnes qui, depuis le «rajeunissement» de l'année dernière, œuvrent en faveur de la Caisse de secours, d'autant plus que la gestion de fortune ne s'est pas simplifiée depuis le début 2001. Toutes ces mutations ont été maîtrisées aisément par le président en charge. Nous ne pouvons que nous en réjouir tant il est vrai qu'il est très important de pouvoir travailler dans un climat harmonieux facilitant la prise de décisions et réduisant les tracasseries administratives, contribuant aussi à abaisser les coûts.

\section{Le Conseil de fondation de la Caisse de secours des médecins suisses: \\ Dr W. Zutter, président Dr L. Heuss, membre du Comité central de la FMH \\ Dr U. Leibundgut, trésorier}

Adresse du président du Conseil de fondation: Dr W. Zutter, Centre de soins ambulatoires Wiesendamm, Wiesendamm 22, 4057 Bâle.

CCP de la Caisse de secours des médecins suisses: 40-644-3 Bâle. 